\title{
Glaeser, Edward (2011): Triumph of the City: How Our Greatest Invention Makes Us Richer, Smarter, Greener, Healthier and Happier
}

\author{
New York: Penguin Press. 338 S.
}

\author{
Eberhard von Einem
}

Eingegangen: 19. Dezember 2012 / Angenommen: 30. Januar 2013 / Online publiziert: 2. April 2013

(C) Springer-Verlag Berlin Heidelberg 2013

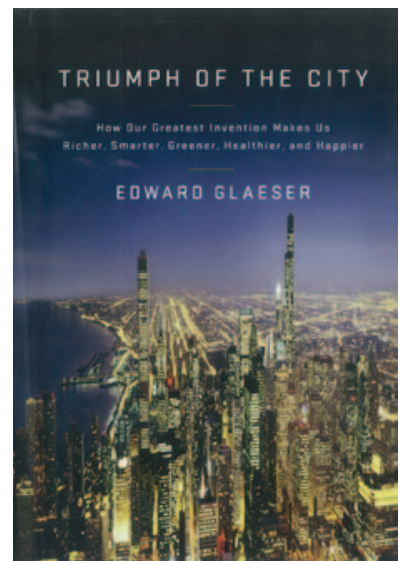

Seit Jane Jacobs' "Death and Life of Great American Cities“ (Jacobs 1961) wurde in vielen Veröffentlichungen beschrieben und wissenschaftlich analysiert, welche Kräfte die unterschiedlichen Entwicklungen von Städten prägen, sei es in Nordamerika, in Europa, in Ostasien, Südamerika oder Afrika.

Von Edward Glaeser, Wirtschaftsprofessor an der Kennedy School of Government der Harvard University, erschien 2011 ein Buch mit dem Titel: „Triumph of the City: How our Greatest Invention Makes us Richer, Smarter, Greener, Healthier and Happier", das es innerhalb weniger Wochen

Prof. Dr. E. von Einem $(\varangle)$

Hochschule für Technik und Wirtschaft,

FB Wirtschaftswissenschaften 1 ,

Treskowallee 8, 10318 Berlin, Deutschland

E-Mail: einem@urban-studies-htw-berlin.de

Prof. Dr. E. von Einem

CMS Center for Metropolitan Studies,

Technische Universität Berlin,

Hardenbergstraße 16-18, 10623 Berlin, Deutschland unter die Top 10 der US-Sachbücher schaffte. Das Buch unterscheidet sich von anderen insofern, als der Autor gegen den Mainstream der kritischen Stadtforschung anschreibt und es ihm ein Anliegen ist, die positiven Errungenschaften „der Stadt“ herauszustellen. Er wagt einen umfassenden Wurf, indem er die Dynamiken der Städte (genauer: der Stadtregionen) auf der Suche nach jenen Faktoren abklopft, die in ihrem Zusammenspiel den Niedergang einerseits und den Aufstieg von Städten andererseits kausal erklären.

Edward Glaeser, als Sohn deutscher Einwanderer in New York aufgewachsen, hat sich in der Fachwelt seit etwa 15 Jahren als Wirtschaftswissenschaftler mit dem Spezialgebiet Stadt- und Regionalökonomie, vor allem durch seine Human Capital Theorie einen Namen gemacht. In zahlreichen Papieren hat er mit den in seiner Disziplin dominierenden statistischen und ökonometrischen Methoden einerseits sowie mit historischen Analysen andererseits die Bedeutung regional verfügbaren Wissens und lokaler Kompetenzen als wichtigsten Faktoren zur Erklärung des Auf und $\mathrm{Ab}$ von Städten herausgearbeitet. Seine Aufsätze sind anspruchsvoll und gelegentlich nur schwer zu verstehen, da sie - wie unter Ökonomen üblich - auf komplexen mathematischen Modellen basieren. Zusammen mit seinen Mitarbeitern veröffentlichte er seine Papiere in der Regel zunächst in den höchst angesehen Working Papers des Harvard Institute of Economic Research oder des National Bureau of Economic Research und später dann in einschlägigen Fachzeitschriften. Damit hat er kumulativ im Laufe der 2000er Jahre belastbare Nachweise dafür erbracht, dass öffentliche und private Investitionen in Ausbildung und Erziehung überproportional die Produktivität, das Einkommen, die Einwohnerzahlen und die Wohlfahrt steigern und zwar sowohl auf nationaler Ebene wie auch auf der Ebene von Städten und Regionen. Beispielsweise fand er heraus, dass jedes zusätzliche Ausbildungsjahr zu einer Erhöhung des regio- 
nalen Brutto-Inlandsprodukts um nicht weniger als 30\% führt (Seite 28). Die Entwicklung von Städten deutet er als mittelbare Folge der geographischen Verteilung öffentlicher und privater Investitionen in Ausbildung und Erziehung: Je höher der Bevölkerungsanteil der College Graduates in einer Stadtregion, seien es Absolventen lokaler Hochschulen oder zugewanderte ausgebildete oder wissenshungrige Talente aus dem In- und Ausland, desto besser stehen diese Städte im globalen Wettbewerb da und desto wahrscheinlicher gelingt es ihnen, Strukturkrisen zu überwinden. Detroit, Cleveland, Buffalo und andere Städte des „Rust Belts“ schaffen es vor allem wegen ihrer geringen Anteile an Hochqualifizierten nicht, den Niedergang aufzuhalten oder gar umzukehren. Die hohen Quoten an Arbeitslosen bauen sich nicht ab, weil die Hochqualifizierten fehlen, die als Katalysatoren indirekt auch Arbeitsplätze für Geringqualifizierte induzieren. Dagegen ist es New York und Boston im Verlauf ihrer 400jährigen Geschichte gleich mehrfach gelungen, sich aus dem Würgegriff temporärer Krisen zu befreien, zuletzt zwischen 1975 und 1980, und sich jeweils neu ,zu erfinden'. Gelungen ist dies vor allem dank der Dichte ihrer Hochschulen, ihren weit überdurchschnittlichen Anteilen an Hochqualifizierten unter allen Erwerbstätigen und ihrer breite Erfahrungen vermittelnden Arbeitsmärkte sowie ihrer historisch belegten Attraktivität für die Zuwanderung von Hochqualifizierten.

Die bisherigen Aufsätze Glaesers erreichten selten eine breite Leserschaft. Das mag den Anstoß dazu gegeben haben, dass er sich entschloss - wie nicht wenige Wissenschaftler vor ihm -, einmal ein populärwissenschaftliches Buch zu schreiben, um seine Thesen und Befunde einer größeren Leserschaft, auch in der Politik, nahezubringen. Sein Buch sollte deshalb als synthetische, allgemein verständliche Kompilation seiner bisherigen Forschungen gelesen werden, auch wenn diese nicht im Einzelnen immer mit Fußnoten belegt sind. Das bedeutet Gewinn und Gefahr zugleich, denn es fällt nicht immer leicht, Glaesers Argumenten zu folgen; vielleicht muss man sogar sagen, dass manche von ihnen ohne die Lektüre seiner früheren, aber im Buch nur kursorisch benannten und dort nicht weiter referierten, Aufsätze und Arbeitspapiere, nicht in ihrer vollen Bedeutung erfassbar sind. In seinem hier rezensierten Buch verzichtet Glaeser nahezu vollständig auf statistische Belege und auf seine ansonsten eleganten und bestechenden Beweisführungen, indem er eher auf illustrierende persönliche - damit aber leider nicht generalisierbare - Erlebnisse, Eindrücke und Beschreibungen abstellt.

Das Buch hat seine Stärke darin, dass sich Glaeser als Autor mit weitem Horizont präsentiert, der ohne Scheu Anleihen bei den Nachbardisziplinen macht. Belesen und ausgestattet mit historischem Hintergrundwissen, zieht er seine Argumentationslinien zur Stadtentwicklung, angefangen von der Antike bis zu den Megacities des globalen
21. Jahrhunderts, stets um die Frage kreisend: Was macht Städte erfolgreich oder eben auch nicht erfolgreich? Seine aktuellen Erfolgsbeispiele beziehen sich in erster Linie auf wissensbasierte Großstadtregionen der westlichen Welt, die technische, organisatorische, kulturelle und politische Innovationen hervorbringen. Er zitiert nicht nur New York, Boston, Vancouver, Minneapolis und San Francisco/Silicon Valley, sondern auch Tokio, Hongkong, Singapur, London, Paris und Mailand. Seine These, auf die er in Varianten immer wieder zurückkommt, lautet: Stadtregionen, in denen sich das ,Humankapital' konzentriert, sind die Vorreiter in der Krisenbewältigung sowie im internationalen Wettbewerb um Innovationen. Sie sind dies weniger wegen ihres Städtebaus, sondern eher wegen ihrer guten Schulen und Forschungsuniversitäten, aber auch wegen ihrer diversifizierten Arbeitsmärkte, wegen ihrer hohen kulturellen Lebensqualität und wegen ihrer ausgebauten Verkehrsinfrastruktur einschließlich internationaler Flughäfen und öffentlicher Verkehrsmittel sowie - nicht zu vergessen - wegen offener, liberaler Einwanderungsgesetze und toleranter lokaler Milieus.

Dem Leser fallen - trotz anderer Diktion - schon bald die Parallelen zu Richard Florida auf, der vor 10 Jahren mit seiner „Talent-Technology-Tolerance“-These die Stadtdebatte nachhaltig und kontrovers beflügelte. In der Tat hatte Glaeser schon 2005 die vermutlich fundierteste aller Kritiken zu Richard Floridas Buch über die „Creative Class“ (2002) formuliert. Im Kern durchaus zustimmend, hatte er - in aller akademischen Fairness - seinem Kollegen entgegengehalten, dass sich dessen „Kreative“, die als notwendiges Ferment des gesellschaftlichen und wirtschaftlichen Wandels zu begreifen sind, letztlich als Spezies unter seine weit gefasste „Human Capital-Theorie“ subsummieren lassen. Ähnlich ist auch Glaesers Forschung zu verstehen, wenn er den Unternehmer wissensbasierter Neugründungen in seine „Human Capital-Theorie“ eingliedern möchte, indem er sich fragt, was ein Unternehmer an gebündelten Top-Qualifikationen benötigt, wenn er sich erfolgreich im globalen Markt positionieren möchte. Da dieser ein komplexes Kompetenzprofil aufweisen sollte, stellt Glaeser heraus, dass die Metropolen der westlichen Welt die besten Voraussetzungen bieten, dieses Bündel an Qualifikationen zu vervollständigen. Dabei argumentiert er, dass ein potenzieller Jungunternehmer seine kombinierten Kompetenzen nur teilweise über formale Wege der Berufs- und Hochschulausbildung gewinnt, sondern zusätzlich über berufliche Erfahrungen on the job erwirbt, wie sie diversifizierte großstädtische Arbeitsmärkte am besten vermitteln: soziale Kompetenzen, Netzwerkbeziehungen und die unbedingte Besessenheit, selbst gesteckte Ziele zu erreichen.

Dennoch, das Buch hat leider auch Schwächen. Glaeser überzieht zuweilen und verlässt den Diskurs des abwägenden Wissenschaftlers, insbesondere wenn er die Grenzen 
seines engeren Fachgebiets verlässt, aber auch, wenn er sich im Schlusskapitel an die Politik wendet. Glaeser neigt dazu, seine Kritiker, die er auf der Seite der ewigen ,Stadtpessimisten' vermutet, mit kernigen, aber nicht immer belegten und deshalb wenig überzeugenden Sätzen zu belehren. Er skizziert - historisch mag er Recht haben - Städte als großartige Leistung der Menschheit, weil sie sich über Jahrhunderte als Motoren für Inventionen und gesellschaftliche Veränderung bewährt und - über die Zeit - Wohlstand generiert haben. Stirnrunzeln lösen jedoch zuweilen seine Verallgemeinerungen individueller Beispiele aus, ganz zu schweigen von seinen apodiktisch klingenden, auf seine Autorität als Harvard-Professor gebauten, Urteilen, die zuweilen als Behauptungen stehen bleiben oder ins Banale abgleiten. Um nicht missverstanden zu werden: Das Buch ist unbedingt lesenswert, aber es kommen Zweifel auf, wenn sich Glaeser einseitig bemüht, Verständnis dafür zu wecken, dass die Gründe für städtischen Niedergang angeblich überall auf der Welt die gleichen seien und er als Beleg hierfür beispielsweise Detroit mit Leipzig vergleicht (Seite 223), während er für den Aufstieg von Städten darauf besteht, dass dieser viele Varianten habe.

Ein Thema, zu dem sich der Leser eine intensivere Erörterung gewünscht hätte, sind die Auswirkungen der digitalen Revolution auf den Zusammenhalt der Städte. Nicht etwa, dass Glaeser diesen Punkt übergeht, er argumentiert, leider ohne Beweise anzuführen, dass der persönliche Austausch von Informationen und Wissen von Angesicht zu Angesicht - trotz Internet und Datenbanken - in dicht bebauten Städten auch im 21. Jahrhundert seine Bedeutung behält und der Austausch von Wissen durch die elektronischen Medien nur teilweise substituierbar ist. Parallel nehmen - so Glaeser - die face-to-face-Kontakte sogar weiter zu. Das wüsste man gern genauer, denn die informationstechnischen Entwicklungen dürften für die Entwicklung der Städte im 21. Jahrhundert einer der entscheidenden Punkte sein. Glaeser verzichtet hierzu leider auf eine abgewogene Auseinandersetzung mit gegenteiligen Thesen (Seiten 37/38 und 248), etwa mit Thomas Friedmans einflussreichem Buch „The World is Flat" (Friedman 2005). Beide, Glaeser wie Friedman, beschreiben den Aufstieg von Bangalore (Indien), weshalb sich ein Vergleich der Thesen angeboten hätte. Glaeser beschränkt sich aber darauf zu wiederholen, wie sehr die Städte vom lokalen ,Humankapital` abhängen, das den persönlichen Austausch von Informationen und wechselseitige Anregungen begünstigt.

Glaeser ist ein vehementer Befürworter von Hochhäusern. Die dichte Stadt, das Wohnen in der dritten Dimension, ist sein Ideal und verraten seine New Yorker Herkunft, nicht zuletzt, weil sie helfen, die Zahl der Autos zu reduzieren und den Klimawandel bremsen. Dass sich die Suburbs auflösen, hält er für unwahrscheinlich. Schrumpfende Städte sind für ihn mit einem Stigma behaftet, wachsende Städte dagegen, vor allem solche, die talentierte, arbeitsbesessene und aufstiegsorientierte $\mathrm{Zu}$ - und Einwanderer anziehen und in ihre Stadtgesellschaften integrieren, sind für ihn das Tor für Erfolg und Wohlstand. Für die Kehrseite, für städtische Armut, Marginalisierung, Slums und Verfall hat er nur am Rande ein Auge. Er ist ein Skeptiker direkter Stadtsanierungs- und sozialer Hilfsprogramme. Er empfiehlt stattdessen, durchaus plausibel, aber dann doch zu kurz greifend, dass in der Bildungspolitik der Schlüssel zum (Wieder-) Aufstieg einer Stadt zu suchen sei, da diese dann mittelbar auch denjenigen zugutekomme, die ohne Bildung chancenlos bleiben. Hier schimmert das in Amerika verbreitete Vorurteil durch, ein jeder könne es durch eigene Anstrengungen schaffen. Eine These, die Glaeser im Schlusskapitel als wichtigste Empfehlung an die Adresse der Politik bereithält, ist: Die Zukunft der Stadt hängt am Faden der Bildungspolitik. Sie dominiert die Stadtpolitik des 21. Jahrhunderts.

\section{Literatur}

Florida, R. (2002): The rise of the creative class and how it's transforming work, leisure, community and everyday life. New York.

Friedman, T. (2005): The world is flat: A brief history of the twentyfirst century. New York.

Glaeser, E. (2005): Review of Richard Florida's The Rise of the Creative Class. In: Regional Science and Urban Economics 35, 5, 593-596.

Jacobs, J. (1961): The death and life of great American cities. New York. 